\title{
Currículo y justicia social: un abordaje desde la interculturalidad
}

\author{
Curriculum and social justice: An approach from interculturality
}

Fausto Fabricio Quichimbo Saquichagua ${ }^{1}$ Nelly Mercedes Mendez Carchi ${ }^{2}$

\section{Resumen}

La interculturalidad en el Ecuador en los últimos años tomó mayor fuerza, generó un amplio debate a nivel discursivo y práctico sobre las diferentes aplicaciones que giran en torno a este concepto polisémico. En este contexto, una serie de directrices y documentos legales - Plan de Desarrollo, Ley Orgánica de Educación Intercultural, Acuerdos Ministeriales - permiten incorporar la interculturalidad en el sistema educativo del país. Así, se visibiliza en los currículos de Educación Intercultural Bilingüe y la etnoeducación. Estas propuestas se trazan en términos de justicia cognitiva y justicia social desde el enfoque de una metodología cualitativa de carácter hermenéutica al analizar los documentos y textos. En este sentido, el presente ensayo discute y reflexiona el aporte de la interculturalidad para la construcción de sociedades con justicia social y como principio de toda la sociedad, así como la relevancia de los contenidos que nos permiten valorar saberes de las diferentes manifestaciones cognitivas, históricas, culturales y lingüísticas del país. De ahí, emerge la necesidad de comprender los currículos desde la Filosofía de la Liberación y la descolonización del saber.

Palabras clave: Educación Intercultural Bilingüe; etnoeducación; interculturalidad; justicia social.

\section{Abstract}

Interculturality in Ecuador in recent years has generated belligerence and a broad debate at a discursive and practical level around this polysemic concept. In this context, a series of guidelines and legal documents - Development Plan, Organic Law of Intercultural Education, and Ministerial Agreements - allow the incorporation of interculturality into the country's educational system. Thus, it is visible in the curricula of Intercultural Bilingual Education and ethno - education. Proposals drawn in terms of cognitive and social justice from a qualitative hermeneutical methodology when analyzing documents and texts. This essay reflects on the contribution of interculturality to the construction of societies with social justice and as a principle of the whole

\footnotetext{
1 Magíster en Educación, mención Educación Intercultural. Técnico docente de la Universidad de Cuenca, Ecuador fabricio.quichimbo@ ucuenca.edu.ec: https://orcid.org/oooo-0oo1-7066-5655

2 Licenciada en Ciencias de la Educación en Historia y Geografía. Investigadora independiente, nelly.mendez@ucuenca.edu.ec: https://orcid.org/oooo-0oo2-2186-2879
}

Recibido: 13/08/2020 - Aprobado: 05/10/2020 
society, thus the relevance of the contents that allow us to assess knowledge of the different cognitive, historical, cultural and linguistic manifestations of the country. Hence, the need to understand curricula from the Philosophy of Liberation and the decolonization of knowledge emerges.

Keywords: Bilingual Intercultural Education; Ethno-education; Interculturality; Social Justice.

\section{Introducción}

La interculturalidad en el Ecuador está ligada a procesos de reivindicación política y social, particularmente de los movimientos sociales de los pueblos indígenas desde la década de los cuarenta, donde la lucha por la tierra, la educación en las propias lenguas ha estado presente en sus demandas. Se incorpora como política de Estado en la Constitución de 1998, constituyéndose en avances significativos dentro de la reflexión sobre interculturalidad a partir del Estado Plurinacional, las circunscripciones territoriales para las nacionalidades y pueblos indígenas, valoración de las lenguas, entre otras. Aunque es en la Constitución del 2008, donde se reconoce al país como un Estado plurinacional e intercultural.

A partir de la Constitución de 2008 una serie de leyes, normativas y políticas giran en torno en interculturalidad; como es el caso de La Ley Orgánica de Educación Intercultural (LOEI), la Ley Orgánica de Educación Superior (LOES). De tal manera que, la interculturalidad en el campo educativo se convierte en tema medular. Además, se encuentra contenida en el Plan Nacional de Desarrollo 2017 - 2021 Toda una Vida a través del objetivo 2. "Afirmar la interculturalidad y plurinacionalidad revalorizando las identidades diversas" que se encuentran relacionados con los objetivos que garantizan una vida digna con iguales oportunidades para todas las personas y con un estado cercano al servicio de la ciudadanía incentivando una sociedad participativa.

Por lo expuesto, este ensayo reflexiona la tesis de que la interculturalidad y el currículo aportan a la construcción de sociedades con justicia social, cuyos núcleos articuladores lo constituyen la filosofía de la liberación y la descolonización del saber. Estos elementos se enmarcan en los currículos de Educación Intercultural Bilingüe (EIB) y la etnoeducación. Hoy en día, el sistema educativo ecuatoriano,de acuerdo a la normativa vigente, cuenta con el currículo nacional, la interculturalidad bilingüe y la etnoeducación. Estos dos últimos se convierten en tema de análisis de este ensayo, en la medida que permiten el reconocimiento de saberes de las diferentes manifestaciones cognitivas, históricas, culturales y lingüísticas de las nacionalidades y pueblos que conviven en el país. 


\section{EDUCACIÓN}

Así, los currículos nacionales interculturales bilingües son oficializados a través del Acuerdo Nro. MINEDUC-MINEDUC-2017-00017-A del 23 de febrero de 2017, elaborados en lenguas de las nacionalidades y en base al Modelo del Sistema de Educación Intercultural Bilingüe (MOSEIB), cuyo proceso pedagógico va desde la Educación Infantil Familiar Comunitaria (EIFC), la Inserción a los Procesos Semióticos (IPS), el Fortalecimiento Cognitivo, Afectivo y Psicomotriz (FCAP), el Desarrollo de Destrezas y Técnicas de Estudio (DDTE) y Procesos de Aprendizaje Investigativo (PAI). Estos currículos se aplican obligatoriamente en el Sistema de Educación Intercultural bilingüe (SEIB).

En cuanto a la etnoeducación, se oficializa con el Acuerdo No. MINEDUC-ME2016-00045-A del 20 de mayo de 2016 y se dirige a los pueblos afroecuatorianos del país cuyo proceso pedagógico se enmarca en el tiempo casa adentro para fortalecer lo propio y casa afuera para enseñar a la sociedad propuesta de Juan García一, propuestas para pensar en un proyecto educativo, desde los pueblos afroecuatorianos del país.

En este contexto, se reflexiona el concepto de interculturalidad. Luego se analizan los currículos de EIB y etnoeducación a partir de la Filosofía de la liberación y la descolonización del saber cómo núcleos articuladores para confluir en una sociedad con justicia social.

\section{Desarrollo}

La interculturalidad es un concepto polisémico que trae consigo una serie de definiciones y debates que, dependen del contexto en el que se desarrolla y el fin de ésta. Puede ser abordada desde cuatro perspectivas: descriptiva, prescriptiva, funcional y crítica. La interculturalidad descriptiva se refiere al conjunto de interrelaciones que se producen entre culturas, en términos de lengua, cultura y etnicidad (Dietz, 2017). Es decir, sólo lo que pasa entre indígenas y afros y vinculada a discursos del indigenismo de los años cincuenta. En palabras de Walsh (2010) el contacto e intercambio entre culturas. No obstante, esta perspectiva oculta los problemas de desigualdad, de dominación, de poder presentes en la sociedad.

La interculturalidad prescriptiva o conocida también como normativa, se enfoca en el desarrollo de medidas de acción afirmativa dirigido particularmente a pueblos y nacionalidades - que se convierten en discriminación positiva (Dietz, 2017). En virtud que, se convierte en instrumentos discursivos enfocados en legitimar procesos de inclusión de los grupos considerados subordinados, quienes deben tener un trato diferente para que puedan formar parte de la sociedad. No obstante, existe una transformación histórica para cambiar las relaciones de opresión, ya que se visibiliza como proyecto y no como una descripción de las cosas (Altmann, 2017). 
La interculturalidad funcional reconoce la diversidad y diferencias culturales, pero se constituye en mecanismo para elevar las desigualdades sociales (Dietz, 2017). En la línea de pensamiento de Walsh (2010) busca promover el diálogo, la convivencia y la tolerancia en una estructura social establecida a partir del reconocimiento de la diversidad y la diferencia. Sin embargo, no se enfoca en las causas que configuran una sociedad de desigualdades y con asimetrías. Es por ello, que debe ser repensada como una herramienta crítica, que cuestione las relaciones de poder en diferentes escalas de la sociedad.

Desde esta mirada, la interculturalidad crítica busca la transformación de las relaciones sociales, las estructuras y las instituciones al interior de la sociedad. Dicho de otro modo, el problema estructural — colonial y racial — para buscar condiciones de estar, ser, pensar, conocer, aprender, sentir y vivir distintas maneras de convivencia (Walsh, 2010). En suma, interculturalidad que se encamina a una transformación estructural para la construcción de una sociedad radicalmente distinta, y no sólo enfocada en pueblos y nacionalidades, sino donde forme parte toda la sociedad.

En este contexto, se reflexiona y se discute el aporte de los currículos de EIB y la etnoeducación, donde la interculturalidad ha estado constantemente presente en los currículos y contenidos de estudio (Vélez, 2019) y su contribución a la construcción de sociedades con justicia social. En el currículo se plasma las intenciones educativas, los lineamientos pedagógicos, las metodologías, los planes de estudio, el perfil de salida que la sociedad requiere y en función de las necesidades del país. En el caso concreto del Ecuador, el diseño curricular considera la visión de un Estado plurinacional e intercultural, atendiendo a las necesidades poblacionales del país rico en tradiciones, culturas y saberes propios.

Los currículos de EIB y la etnoeducación nos permiten pensar desde la diversidad para mejorar la convivencia en los contextos educativos, sociales y culturales. Además de formar sujetos con conciencia histórica, comprometidos con la justicia social, como respuesta a las asimetrías presentes. De ahí que, los currículos contemplan contenidos que se encaminan a la descolonización del saber, permitiendo mostrar historias locales, memoria oral. Contrariamente a los currículos de corte eurocéntrico que imponen una historia universal, donde América Latina y el África no forman parte de la historia (Dusell, 1996). Sin embargo, deben existir puentes de encuentro entre conocimientos propios y occidentales. Coincidimos con Inuca (2017) que se aprendan conocimientos de la vida cotidiana y la cultura propia y de la cultura universal. Esto quiere decir, promover la interacción entre conocimientos científicos y "no científicos", ósea un diálogo de saberes (Sousa, 2010).

También se encaminan a desarrollar competencias que recaen en los campos del saber: saber ser, saber hacer y saber conocer. Así, actuar con responsabilidad y justicia social, -no sólo dentro del contexto educativo, sino en la sociedad-frente a 


\section{EDUCACIÓN}

las injusticias sociales, producto de la discriminación, racismo, xenofobia que siguen latentes hasta la actualidad. De hecho, la colonialidad del poder, del ser y del saber siguen latentes hasta nuestros días, ya que es uno de los constitutivos del patrón mundial del poder capitalista (Quijano, 2000).

Si bien, los currículos de EIB y la etnoeducación se enmarcan en los tres principios de justicia curricular de Connell (1997), intereses de los menos favorecidos, participación y escolarización común, la producción histórica de la igualdad; y se encuentran transversalizados por la interculturalidad, todavía no existe una implementación adecuada. En virtud que, son tomados como currículos paralelos al currículo nacional. Además, están direccionados solo para pueblos y nacionalidades indígenas y pueblo afroecuatoriano, dejando de un lado a la población mestiza, incluyéndose en el sistema, pero excluyendo a la vez. Sumado a que, la interculturalidad es pensada solo en su relación con la educación bilingüe y de los pueblos indígenas. Situación que provoca, una interpretación culturalista y etnicista (Altmann, 2017). Es por ello, que los imaginarios y las prácticas docentes en el aula están asociadas a bailes y trajes típicos, comida, juegos "ancestrales", desvirtuando el concepto de interculturalidad. De ahí, el compromiso como docentes y de toda la sociedad para repensar la interculturalidad desde perspectivas críticas y reflexionar el aporte de los currículos de EIB y la etnoeducación para desarrollar procesos de inclusión, valoración, interacción y respeto, para construir sociedades en el marco de la justicia social.

En la línea de pensamiento de Sousa (2010) la batalla por la justicia social atraviesa por la justicia cognitiva. En este contexto, los currículos de EIB y la etnoeducación se enmarcan en este cometido. Fruto de reclamos de los grupos sociales que han sufrido injusticias, desigualdades y discriminaciones. Por ello, la inclusión de contenidos de los grupos sociales más desfavorecidos que es el caso de los pueblos indígenas y afrodescendientes, nos invitan a desaprender y romper estructuras mentales; y a su vez cuestionarnos ¿con quiénes queremos pensar y para qué?, así, interculturalizar, desde el aula, desde los programas o como práctica de campo (Quichimbo, 2019). Pongamos por caso, en la etnoeducación se quiere que se visibilice que el pueblo afrodescendiente fue esclavizado y no fueron esclavos; aunque pueden parecer situaciones iguales, existe un gran distanciamiento, ya que, la esclavitud pasó por un proceso de institución por parte de los colonizadores, ya que nunca existieron esclavos.

A partir del ejemplo señalado, resulta necesario desaprender y reflexionar qué clase de historia de nuestro pueblo se construyó y se construirá. En virtud que, en la historiografía tradicional es común que sea promovida por los grupos hegemónicos quienes definen y evalúan el pasado en base a sus intereses, donde las voces de los vencidos permanecen ocultas y sobresale en la cúspide la de las clases dominantes. Ejemplo de ello, son los textos de Historia y Ciencias Sociales de nuestro país, que visibiliza "héroes" - que particularmente son de las élites - en los procesos independistas, y nunca se habla de los grupos que estuvieron detrás, quienes con auspicia y 
furor comandaban los batallones. Tal y como es el caso, del rol del pueblo negro en los procesos independistas ocurridos en el continente, que la historia tradicional no cuenta.

Por esta razón, desde la etnoeducación, se trabaja desde las premisas de casa adentro y casa afuera, en el sentido de comprender y entender la historia del pueblo afro. Reflexionado a partir de conceptos teóricos para abordar los posicionamientos políticos, epistemológicos y culturales. Además del papel de los líderes y lideresas afroecuatorianos. Y lucha contra la invisibilización que históricamente han sufrido y se encamina a cambiar la visión racista, estereotipada que se ha construido en torno a este pueblo que le debemos mucho. En palabras de Vera (2017) producto del colonialismo y esclavitud han existido regímenes racializados de representaciones que producen jerarquización, estereotipos y discriminación. En este contexto, la Flosofía de la Liberación y la descolonización se convierten en mecanismos para fomentar un pensamiento académico contrahegemónico desde una perspectiva emancipadora.

De igual manera, el MOSEIB se sustenta desde los principios de respeto y cuidado a nuestra Madre Naturaleza, donde la comunidad educativa -la persona, la familia, la comunidad, los administradores educativos- se convierte en los representantes principales del proceso pedagógico en todas sus unidades de aprendizaje. Tomado en cuenta las lenguas de las nacionalidades, los conocimientos, las prácticas culturales, entre otros. En suma, un modelo de descolonización, de emancipación, de justicia cognitiva y justicia social.

En este contexto se justifica que el MOSEIB y la etnoeducación atraviesa procesos de descolonización del saber, del poder, del ser; en los cuales no existen dicotomías excluyentes, que han señalado que lo pueblos indígenas y pueblos afrodescendientes no tienen cultura ni ciencia, sino folclor y mitos. En esta dirección, nos permite encaminarnos a una pedagogía liberadora y emancipadora para formar seres humanos con sentido crítico y autónomo en el marco del diálogo frontal, respetuoso, equitativo con el fin de superar estigmas sociales de colonialidad, subordinación, supremacía y dominio. Y así, caminar hacia la construcción de sociedades con justicia social.

\section{Conclusiones}

Los currículos desde una perspectiva intercultural deben orientarse a una reflexión flexible, crítica, contextualizada, alineada a las demandas de toda la sociedad y que requiere la educación del siglo XXI. Encaminados al fortalecimiento del proceso educacional intercultural en todos los niveles: primario, secundario y superior. Es por ello, que los educadores y profesionales en educación deben trabajar desde un enfoque intercultural, que trasciende lo étnico y lo cultural; promoviendo dentro de sus contenidos macro, meso y micro curriculares, la construcción de sociedades con justicia social y justicia cognitiva. 
La perspectiva intercultural, constituye el camino para fomentar relaciones equitativas, inclusivas, respetuosas, donde se valoren voces y visiones de los diferentes miembros de la sociedad para mejorar la convivencia. Así, crear espacios de diálogo intercultural para generar una nueva conciencia educativa y social; que cuestione las opresiones de poder a los que son sometidos los diferentes miembros de la sociedad.

EL MOSEIB y la etnoeducación nos invitan a pensar en una transformación educativa desde la Filosofía de la Liberación y la descolonización, con visión a largo plazo, donde se incorpore el reconocimiento de las tradiciones, leyendas, mitos, saberes, sabiduría - del ayer y del hoy - y promueva la interacción de los diferentes grupos que conviven en el país. Sin embargo, no debe direccionarse a determinados grupos, sino a toda la sociedad, ya que se fomenta una exclusión educativa. En esta dirección, los diferentes currículos de EIB, el nacional y la etnoeducación deben articularse en uno solo. Finalmente, mencionar que, la interculturalidad, es una práctica de lucha diaria y constante, porque no es fácil romper con la lógica establecida.

\section{Agradecimiento}

Esta publicación obtuvo el financiamiento de: El Fondo de Asistencia Internacional de los Estudiantes y Académicos Noruegos (SAIH).

\section{Lista de referencias}

Altmann, P. (2017). La interculturalidad entre concepto político y one size fits all. En J. Gómez. Repensar la interculturalidad. Guayaquil: UARTES ediciones (pp. 13-36).

Dietz, G. (2017). Interculturalidad: una aproximación antropológica. Perfiles Educativos, 39(156), 192-207. Recuperado de http://www.scielo.org.mx/scielo. php?pid=So185-26982017000200192\&script=sci_arttext

Dussel, E. (1996). Filosofía de la Liberación. (4ª ed.). Bogotá: Nueva América.

Gómez, J. (2017). (Ed.). Repensar la interculturalidad. Guayaquil: Uartes, ediciones

Inuca, J. (2017). Kawsaypura yachay tinkuy. En J. Gómez. Repensar la interculturalidad. Guayaquil: UARTES EDICIONES. (pp. 37 - 71)

Quichimbo, F. (2019). La comprensión de la interculturalidad en el Ecuador: retos y desafíos. Revista Andína de Educación 2(2), 15-23. Recuperdo de https://revistas. uasb.edu.ec/index.php/ree/article/view/1127 
Quijano, A. (2000). Colonialidad del poder y clasificación social. En Santiago CastroGómez y Ramón Grosfoguel (eds). El giro decolonial. Reflexiones para una diversidad epistémica más allá del capitalismo global. Bogotá: Siglo del Hombre Editores, 2007 (Primera edición en: Festschrift For Immanuel Wallerstein. En Journal of World Systems Research, vol. VI, $N^{o}$ 2, Fall/Winter, pp.342-388. Special Issue. Giovanni Arrighi and Walter L. Goldfrank, eds. Colorado, 2000).

Sousa, B. (2010). Descolonizar el Saber, reinventar el poder. Montevideo: Ediciones Trilce.

Vélez, C. (2009). Trayectoria de la educación intercultural en Ecuador. En A. Mendoza y F. Yánez. (Comps.). Interculturalidad: reflexiones desde la práctica. Quito: UNICEF.

Vera, R. (octubre, 2017). La etnoeducación como posicionamiento político e identitario del pueblo Afroecuatoriano. Revistas Antropológicas del Sur. Año $4 \mathrm{~N}^{\circ} 8$ 2017 Págs. 81 - 103. Recuperado de http://www.revistaantropologiasdelsur.cl/ wp-ontent/uploads/2017/12/1219_vera.pdf

Walsh, C. (2010). Interculturalidad crítica y educación intercultural. En J. Viaña., L, Tapia., \& C. Walsh. Construyendo interculturalidad crítica. Lima: Instituto Internacional de Integración del Convenio Andrés Bello. (pp. 75 - 96). Recuperado de http://www.aulaintercultural.org/IMG/pdf/Interculturalidad_Critica_y_ Educacion_Intercultural1.pdf 